\title{
Dietary diversity in eight Latin American countries: Results from ELANS Study
}

\author{
Georgina Gomez ${ }^{1}$, Regina Mara Fisberg ${ }^{2}$, Agatha Nogueria Previdelli ${ }^{3}$, Irina Kovalskys ${ }^{4}$, \\ Mauro Fisberg ${ }^{5,6}$, Marianella Herrera-Cuenca ${ }^{7}$, Lilia Yadira Cortes Sanabria ${ }^{8}$, \\ Martha Cecilia Yepez ${ }^{9}$, Rossina G. Pareja ${ }^{10}$ and Attilio Rigotti ${ }^{11}$ \\ ${ }^{1}$ University of Cost Rica, San Jose, Costa Rica, \\ ${ }^{2}$ University of Costa Rica, Sao Paulo, Brazil, \\ ${ }^{3}$ Universidade Sao Judas Tadeu, Sao Paulo, Brazil, \\ ${ }^{4}$ International Life Science Institute, Buenos Aires, Argentina, \\ ${ }^{5}$ Instituto Pensi, Sao Paulo, Brazil, \\ ${ }^{6}$ Universidade Federal de Sao Paulo, Sao Paulo, Brazil, \\ ${ }^{7}$ Universidad Central de Venezuela, Caracas, Venezuela, Bolivarian Republic of., \\ ${ }^{8}$ Pontificia Universidad Javeriana, Bogota, Colombia, \\ ${ }^{9}$ Universidad de San Francisco de Quito, Quito, Ecuador, \\ ${ }^{10}$ Instituto de Investigación Nutriciona, Lima, Peru and \\ ${ }^{11}$ Pontificia Universidad Catolica, Santiago, Chile
}

\begin{abstract}
Dietary diversity, define as the number of food items or food groups consumed over a given period of time measured at the household or individual level, is widely recognized as a key dimension of diet quality. This analysis investigated dietary diversity in eight Latin-American countries and its associations with sociodemographic and anthropometric parameters.

Data from the ELANS study conducted in eight Latin-American countries (Argentina, Brazil, Colombia, Costa Rica, Chile, Ecuador, Peru and Venezuela) were analyzed. The ELANS study interviewed 9,218 subjects living in the main cities in each country. Food intake were collected using two 24-hour dietary recalls, following the Multiple Pass Method. For calculation of diet diversity score, only the first 24-hour recall was analyzed. Dietary diversity was assessed at individual level. All food items reported to be consumed during the first 24-hour recall were classified into nine food groups: 1. Cereals, 2. White roots and tubers, 3. Vegetables, 4. Fruits, 5. Meat, poultry and offal, 6. Fish and seafood, 7. Eggs, 8. Pulses, legumes and nuts and 9. Milk and milk products. The selection of these groups was based on the Women's Dietary Diversity Score Projectfood groups classification. Consumption of at least $15 \mathrm{~g}$ of each food group was assigned 1 point or 0 points if consumption was less than $15 \mathrm{~g}$. Thus, the score ranged from a minimum of 0 and a maximum of 9 points. Higher scores indicated higher diversity as more food groups were eaten. Analysis was performed by age group, gender, and socioeconomic level (SES) as well as anthropometric measurements.Mean diet diversity score (DDS) for the whole sample was $4.48 \pm 1.16$, ranging from 0 to 9 points. Men showed significant higher DDS. No difference was observed among age groups. Among countries, Ecuador and Peru showed the highest DDS, $4.88 \pm 1.22$ and $4.82 \pm$ 1.12 points, respectively; while Argentina ( $4.20 \pm 1.13)$ and Venezuela $(4.27 \pm 1.04)$ reported the lowest. We found a statistically significant trend $(\mathrm{p}<0.001)$ to a higher DDS among those subjects in the high socio-economic level. Regarding anthropometric measurements, no differences were found in DDS among different nutritional status or based on waist and neck circumference measures. This study revealed that dietary diversity is limited among Latin American countries regardless of sex, age, socioeconomic level, and nutritional status. Nutritional interventions emphasizing the importance of maximizing dietary diversity should be encouraged to ensure optimum nutritional adequacy within the region
\end{abstract}

\section{Conflict of Interest}

There is no conflict of interest 